\title{
RALBP1/RLIP76 mediates multidrug resistance
}

\author{
KENNETH J. DRAKE, JYOTSANA SINGHAL, SUSHMA YADAV, AALOK NADKAR, \\ CHIRAG PUNGALIYA, SHARAD S. SINGHAL and SANJAY AWASTHI
}

Department of Chemistry and Biochemistry, University of Texas at Arlington, Arlington, TX 76019-0065, USA

Received July 17, 2006; Accepted August 31, 2006

\begin{abstract}
RLIP76/RALBP1 is a multi-specific drug-transporter which can mediate drug-resistance in lung and other cancers, but its ability to mediate multidrug-resistance has not been previously demonstrated in hematological malignancy. Present studies in K562 human myelogenous leukemia show that RALBP1 overexpression confers broad resistance to multiple chemotherapy drugs including cisplatin, melphalan, doxorubicin, daunorubicin, vincristine, vinblastine, vinorelbine, and mitomycin-C. Conversely, inhibition of RALBP1 by polyclonal antibodies causes increased drug-accumulation and increased cytotoxicity. These studies demonstrate the potential utility of targeting RALBP1 in the treatment of leukemia.
\end{abstract}

\section{Introduction}

Multidrug-resistance (MDR), a phenotype exhibited by many cancers typified by resistance to the cytotoxic effects of structurally unrelated cytotoxic agents, remains a major obstacle to the eradication of malignancy with chemotherapy. Numerous cellular biochemical mechanisms have been

Correspondence to: Professor Sanjay Awasthi, Department of Chemistry and Biochemistry, 700 Planetarium Place, CPB Room 351, University of Texas at Arlington, Arlington, TX 76019, USA

E-mail: sawasthi@uta.edu

Abbreviations: MDR, multidrug-resistant phenotype; Pgp, Pglycoprotein ( $m d r-1$ gene product, also referred to as ABCB1); $\mathrm{ABC}$, ATP-binding-cassette family; MRP, multidrug-resistance associated protein, now referred to as MRP1 or ABCC1; ABCG2, BCRP; RALBP1, human gene encoding several splice variants including RLIP76; CDDP, cis-diamminedichloro-platinum II or cisplatin; MEL, melphalan; DOX, doxorubicin; DAU, daunorubicin; VCR, vincristine; COL, colchicine; VBL, vinblastine; VRL, vinorelbine; MMC, mitomycin-C; MCB, monochlorobimane; 4HNE, 4-hydroxy- $t$-2- nonenal; GS-HNE, the glutathione adduct of 4HNE; GS-DHN, 2-glutathionyl-1,4-dihydroxy nonane formed through reduction of the terminal aldehyde to alcohol catalyzed by aldose reductase; GGT, $\gamma$-glutamyltranspeptidase; Hsf-1, transcription factor, heat-shock factor-1

Key words: RALBP1, RLIP76, K562, MDR, transport identified as contributors to the MDR phenotype, though the prototypical MDR is most frequently associated with a decrease in cellular accumulation of drug due to active energy-dependent efflux of drugs or metabolites (1-4). Accumulation defective MDR is mediated by a diverse array of transporter proteins, some well-characterized as transporters and other for which there is indirect evidence for transporter function.

Pgp ( $m d r-1$ gene product, ABCB 1$)$ was the first identified drug-efflux pump shown to mediate resistance to a broad variety of weakly basic amphiphilic xenobiotic compounds through their ATP-dependent efflux $(5,6)$. MRP (ABCC1) was subsequently identified as another related transporter protein also capable of mediating MDR through drug-efflux (6-8). Subsequently, at least 46 other ABC family proteins have been identified, some with demonstrated transport activities comparable with $\mathrm{ABCB} 1$ or $\mathrm{ABCC} 1$, and others with presumed transport function on the basis of homology $(3,6-8)$. The most remarkable difference in substrate specificity between $\mathrm{ABCB} 1$ and $\mathrm{ABCC} 1$ is the ability of the latter to also transport a variety of anionic metabolites such as glutathioneelectrophile thioethers conjugates (GS-E). GS-E, formed through the catalytic action of GSTs in the first and rateregulating step of the mercapturic acid pathway for biotransformation of endogenous and exogenous electrophilic toxins, can be transformed into other more reactive and toxic compounds in cells $(9,10)$. Endogenous lipid peroxidation by-products such as the potent pro-apoptotic compound, 4hydroxynonenal (4HNE), are also metabolized to GS-E (GS-HNE). Inhibiting the efflux of endogenous GS-E inhibits metabolism of $4 \mathrm{HNE}$, increasing the concentration of this reactive lipid aldehyde, and triggering apoptosis $(11,12)$. Under certain conditions, GS-HNE can also be reduced by aldose reductase to GS-DHN which is a key signaling intermediate in mitogenic signaling (13). Cellular accumulation of GS-E is thus a very closely regulated process, and is dependent on the removal of these compounds through active efflux across the plasma membrane, where $\gamma$-glutamyl transpeptidase (GGT) located on the outer plasma membrane leaflet catalyzes the next step of mercapturic acid biosynthesis (9).

Although a number of ABC-transporters can mediate the efflux of GS-E, recent studies of function in knockout animals show that the bulk of efflux of model substrate allocrites such as DOX and GS-E is actually mediated by a non-ABC transporter, RALBP1, a low-affinity high-capacity transporter which has substrate specificity similar to but not identical to ABCC1 and ABCG2 (14). RALBP1 is a stressresponsive, stress-protective ATP-dependent transporter of 
GS-E and xenobiotic toxins such as chemotherapy agents (15-22). Knockout of the mouse homolog of RALBP1 (referred to as Ralbp1) causes $\sim 80 \%$ loss of transport capacity for DOX and GS-E, and markedly increased sensitivity to stress, xenobiotics, as well as ionizing radiation (14). It serves an anti-apoptotic function by preventing accumulation of endogenously derived GS-E. Endogenous GS-E, which form as a result of oxidant or radiant stress, compete for efflux with a number of chemotherapy drugs including DOX, DAU, COL, VCR, VBL, and VRL, GS-E derived from alkylating agents or platinum coordinates which are known substrates for transport by RALBP1 (15-24).

Our recent studies demonstrate that this mechanism plays a key anti-apoptotic role which is of central importance in malignant cells, but is not essential in non-malignant cells. Because of this, depletion or inhibition of RALBP1 in mouse syngeneic as well as human xenograft tumor models causes complete and durable responses without affecting weight gain or causing other toxicities (22). These observations place RALBP1 as a central anti-apoptotic effector mechanism, an assertion validated by studies of others who have independently identified the function of RALBP1 as being directly linked as an effector in multiple signaling pathways critical in malignancy including Ras, Ral, cdc2, cdc42, Hsf-1, Src, EGF, TGFß, Akt, and insulin (25-28).

Our previous studies have focused on the role of RALBP1 as an anti-apoptotic GS-E and drug-transporter in solid tumors including melanoma, lung, prostate, and ovary (22). Since RALBP1 is also expressed in cell lines derived from hematological malignancies (29), we have directly addressed the question of whether RALBP1 can play a role in mediating multidrug resistance in K562 human erythroleukemia cells in the present study. The results presented here indicate that RALBP1 can also function as a multidrug resistance mediating protein in K562 cells.

\section{Materials and methods}

Reagents. Reagents were obtained from the following; MEL was purchased from Sigma-Aldrich Company (St. Louis, MO), VRL from Gensia-Sicor Pharmaceutical Company (Irvine, CA), DOX from Adria Laboratories (Columbus, $\mathrm{OH})$, DAU from Weyth Laboratories (Philadelphia, PA), VCR from Mayne Pharma (Mulgran, Australia), VBL from American Pharmaceutical Partners (Los Angeles, CA), and CDDP and MMC from Bristol-Meyers Squibb (Princeton, $\mathrm{NJ})$. Reagents for preparing culture media were obtained from DIFCO Laboratories (Detroit, MI). Cell culture reagents were obtained from Life Technologies Incorporated (Grand Island, NY). Chemical reagents and horseradish peroxidasecoupled goat anti-rabbit antibodies were obtained from SigmaAldrich Chemical Company. Rabbit anti-human rec-RALBP1 polyclonal antibodies were made and purified according to a method previously described by us $(15,29)$. DE-52 (DEAE cellulose) anion exchanger was purchased from Whatman International Limited (Maidstone, UK). Bio Beads (SM-2 adsorbent) and Chelex-100 resin was purchased from BioRad Laboratories (Hercules, CA). DNPSG-affinity resin for RALBP1 protein purification was prepared by a method previously reported by us $(15,29)$.
Cell culture. Human erythroleukemia cell line K562 was obtained from American Type Culture Collection, Manassas, $\mathrm{VA}$, and cultured at $37^{\circ} \mathrm{C}$ in a $5 \% \mathrm{CO}_{2}$ atmosphere in RPMI1640 medium containing $2 \mathrm{mM}$ Glutamine, $10 \%$ (v/v) fetal bovine serum (FBS) heat-inactivated $1 \%(\mathrm{v} / \mathrm{v})$ penicillin/ streptomycin solution. Cultures were maintained between 100,000 and 1,000,000 cells $/ \mathrm{ml}$. Cells with transfection contained an additional antibiotic Geneticin (G-418 $300 \mu \mathrm{g} / \mathrm{ml})$.

Transfection of K562. K562 cells were transfected with pcDNA3.1-RALBP1 using a Lipofectamine 2000 transfection reagent kit (Invitrogen), and controls were transfected with the empty vector alone as previously described $(15,20)$. RNA was extracted and purification was carried out using an RNeasy kit (Qiagen). Expression of RALBP1 mRNA in K562 cells was evaluated by RT-PCR analysis. Overexpression of RALBP1 protein in K562 cells was evaluated by applying $200 \mu \mathrm{g}$ aliquots of crude membrane extract to SDS-PAGE, followed by Western blot analyses using anti-RALBP1 IgG as a primary antibody. Fold induction of RALBP1 was quantified by scanning densitometry. Western blots were developed using the HRP method (Bio-Rad). For purification of RALBP1 from cells, DNPSG-Sepharose affinity chromatography purification was carried out from $1 \times 10^{8}$ cells from control and each transfected cell type. Purified protein (10 $\mu \mathrm{g}$ protein) was applied to SDS-PAGE, and subjected to Western blot analyses towards anti-RALBP1 antibodies.

Drug sensitivity assay. Viable trypan-blue excluding cell density was determined by hemocytometer, and $2 \times 10^{4}$ cells were seeded into each well of a 96-well plate in $200 \mu 1$ RPMI medium. After 24-h incubation, $40-\mu 1$ aliquots of drugs (CDDP, MEL, DOX, DAU, VCR, VBL, VRL, or MMC) freshly diluted in medium were added to achieve final concentrations between 10 and $10,000 \mathrm{nM}$ to 8 replicate wells. After 72 -h incubation, $20 \mu \mathrm{l}(5 \mathrm{mg} / \mathrm{ml}$ stock) MTT was added to each well and incubated for $2 \mathrm{~h}$ at $37^{\circ} \mathrm{C}$. The plates were centrifuged and medium was removed. Formazan dye trapped in cells was dissolved by addition of $100 \mu 1$ DMSO with gentle shaking for $2 \mathrm{~h}$ at room temperature, followed by measurement of absorbance at $570 \mathrm{~nm}$. $\mathrm{IC}_{50}$ values were obtained using nonlinear regression to fit a modified Hill equation (30).

Drug uptake studies. Cells growing in log phase were collected and washed with PBS, and aliquots containing $5 \times 10^{6}$ cells were diluted in fresh RPMI medium containing $\left[{ }^{3} \mathrm{H}\right]-\mathrm{VRL}$ $(0.1 \mu \mathrm{M})$ or $\left[{ }^{14} \mathrm{C}\right]$-DOX $(3.6 \mu \mathrm{M})$ and then added to the medium and allowed to incubate for a predetermined time period (5, $10,20$, and $30 \mathrm{~min})$ at $37^{\circ} \mathrm{C}$. Drug uptake was stopped by rapid cooling on ice. Cells were centrifuged at $2000 \mathrm{x}$ g for 5 min at $4^{\circ} \mathrm{C}$ and supernatant was decanted. Radioactivity was determined in the cell pellet after rinsing twice with ice-cold PBS using the Beckman LS-230 scintillation counter.

Drug efflux studies. Cells were collected and rinsed with PBS; aliquots containing $5 \times 10^{6}$ cells were suspended in fresh medium. After overnight incubation, the cells were centrifuged, pelleted, and re-suspended in $90 \mu 1$ medium. [ $\left.{ }^{3} \mathrm{H}\right]-\mathrm{VRL}$ $(0.1 \mu \mathrm{M})$ or $\left[{ }^{14} \mathrm{C}\right]-\mathrm{DOX}(3.6 \mu \mathrm{M})$ was then added to the medium and allowed to incubate for $60 \mathrm{~min}$ at $37^{\circ} \mathrm{C}$. Cells 

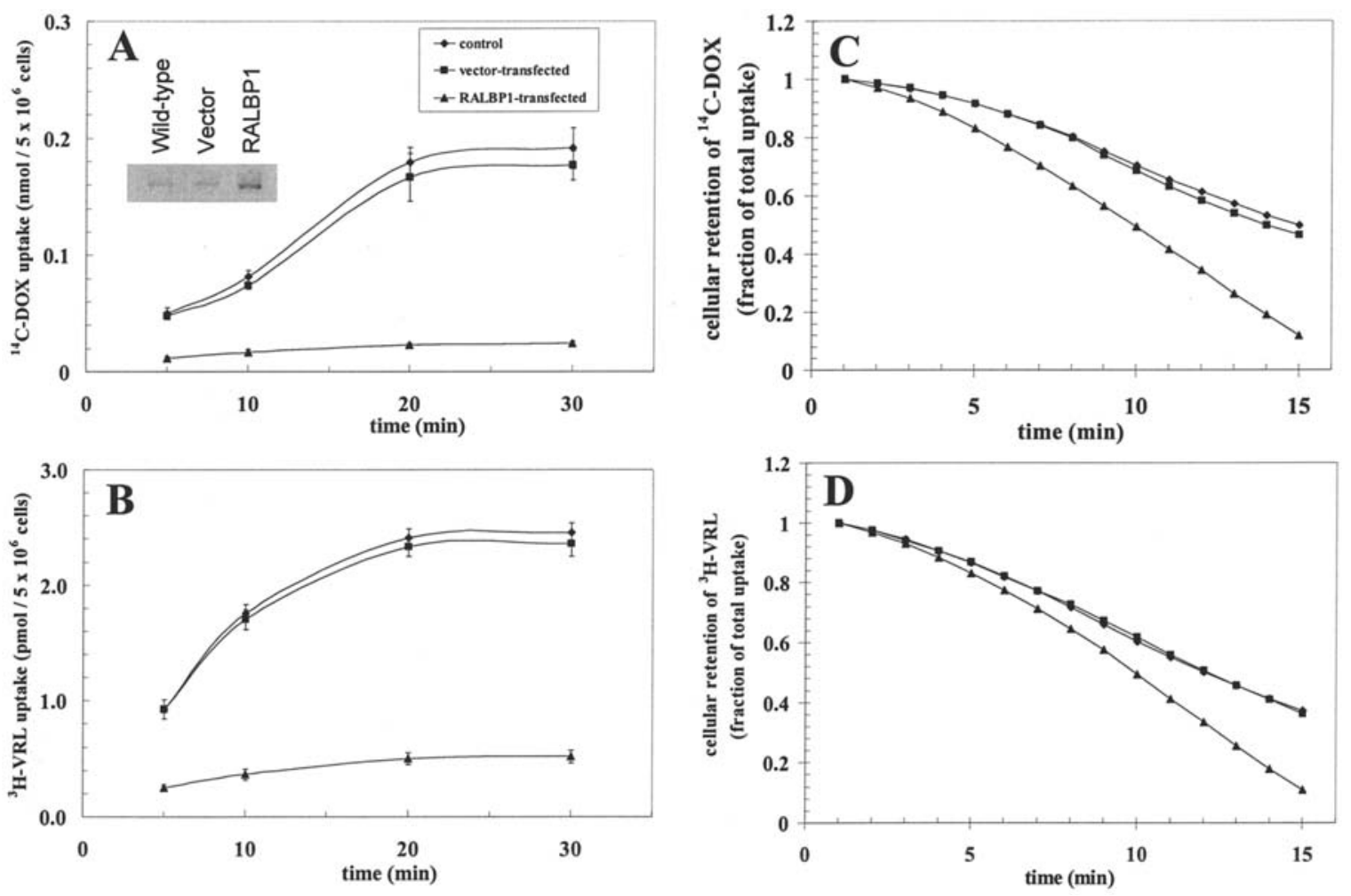

Figure 1. Effect of RALBP1 overexpression on DOX and VRL uptake and efflux in K562 erythroleukemia. The overexpression of RALBP1 in K562 cells was confirmed by Western blot analyses using anti-RALBP1 IgG (A, inset). The rate of cellular accumulation of [ $\left.{ }^{14} \mathrm{C}\right]-\mathrm{DOX}$ and $\left[{ }^{3} \mathrm{H}\right]-\mathrm{VRL}$ was evaluated using

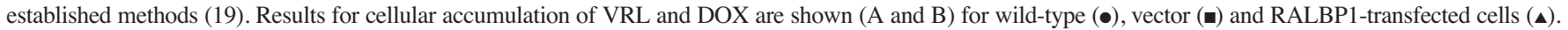
The efflux of DOX was determined by integration of measurements of DOX or VRL appearing in the medium as well as the residual at the end of the experiment to obtain cellular drug-level and efflux rates as per results of a previously published study (19) (C and D). All studies were performed in triplicate, with means and SD presented above.

were centrifuged at $300 \mathrm{x} \mathrm{g}$ for $5 \mathrm{~min}$, after which supernatant was removed completely and the cell pellet was washed twice with PBS. The pellet was immediately re-suspended in $1 \mathrm{ml}$ of PBS; $50-\mu 1$ aliquots (clear supernatant) were removed every minute for $15 \mathrm{~min}$ and added to vials containing scintillation fluid. The residual radioactivity in remaining PBS and cells at the end of 15 min was counted and back-addition was used to determine the cell-associated drug at each time-point as described previously (19).

Cellular accumulation of MCB and DOX by fluorescence microscopy. Cells growing in suspension culture at a density of $5 \times 10^{5}$ cells $/ \mathrm{ml}$ were treated with either $50 \mu \mathrm{M} \mathrm{MCB}, 10 \mu \mathrm{M}$ $\mathrm{DOX}$, or both for $20 \mathrm{~min}$ at $37^{\circ} \mathrm{C}$, rinsed with $\mathrm{PBS}$, and cytospun on to glass slides using a Cytopro Cytocentrifuge (Wescor, Inc., UT). Subsequently, the slides were rinsed with PBS, fixed with $4 \%$ paraformaldehyde in PBS, and covered with glass cover-slips using Vectashield mounting medium for fluorescence (Vector Laboratories, CA). Fluorescence photomicrographs were taken using a LEICA DMLB (Germany) fluorescence microscope at magnification $x 400$. The excitation and emission filters used were 380 and $470 \mathrm{~nm}$ respectively for $\mathrm{MCB}$, and 495 and $550 \mathrm{~nm}$ respectively for DOX.

\section{Results and discussion}

Expression of RALBP1 in K562. Stable overexpression of RALBP1 was achieved in K562 cells as indicated by Western blotting against anti-RALBP1 antibody (Fig. 1A, inset). RALBP1 protein level was unaffected by empty vector transfection. Quantifying RALBP1 protein by ELISA assay (20) showed a 3.8-fold increase in RALBP1 protein in overexpressing cells as compared with wild-type or empty vector transfected cells. This corresponded to a 2.7-fold greater RALBP1 mRNA content by RT-PCR using the forward (nt 1496-1515) and reverse (nt 1948-1968) primers. RALBP1 expression did not cause significant morphological alteration, but the growth rate was increased as evidenced by a reduction in doubling time by $20 \%$.

Effect of RALBPI overexpression on drug uptake and efflux. The uptake and efflux of $\left[{ }^{3} \mathrm{H}\right]-\mathrm{VRL}$ and $\left[{ }^{14} \mathrm{C}\right]-\mathrm{DOX}$ was compared between wild-type, empty-vector, or RALBP1 transfected K562 cells using established methods (19). A marked decrease in total uptake of both drugs was observed in cells overexpressing RALBP1 (Fig. 1A and B). The efflux of both drugs from cells loaded with either drug was greater with RALBP1-overexpression (Fig. 1C and D). The emptyvector control was unaffected.

The greater cellular accumulation of DOX was confirmed by fluorescence microscopy. The effect of RALBP1 on GS-E accumulation was also examined using monochlorobimane (MCB) which yields a fluorescent glutathione conjugate $(31,32)$. As with DOX, MCB accumulation in cells was reduced significantly in RALBP1 overexpressing cells (Fig. 2). Fluorescence micrographs taken of cells loaded with both 


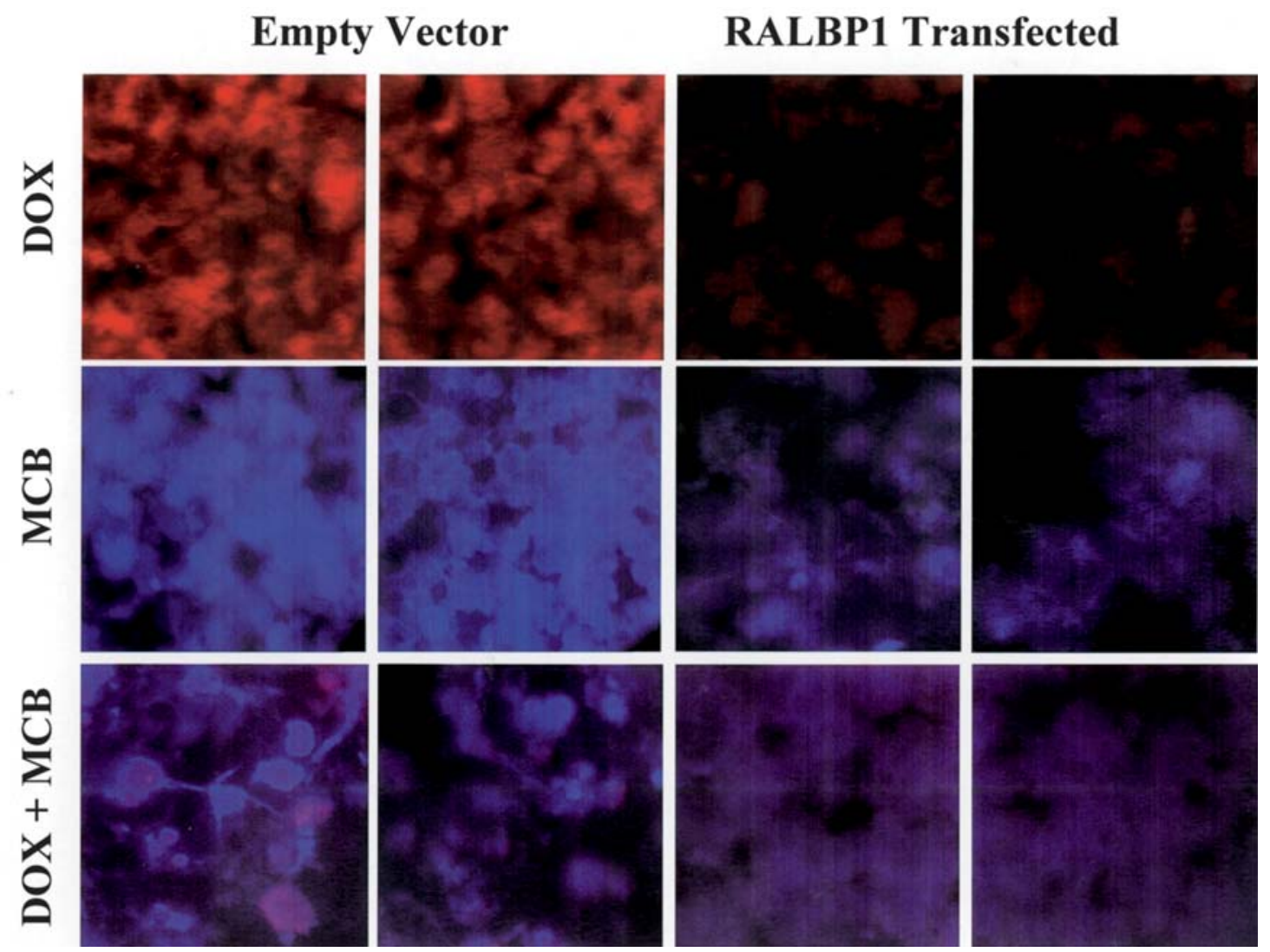

Figure 2. Effect of RALBP1 overexpression on DOX and MCB uptake. K562 cells growing in suspension culture at a density of $5 \times 10^{5}$ cells $/ \mathrm{ml}$ were treated with either $50 \mu \mathrm{M} \mathrm{MCB}, 10 \mu \mathrm{M}$ DOX, or both for $20 \mathrm{~min}$ at $37^{\circ} \mathrm{C}$, rinsed with PBS, and cytospun onto glass slides. The excitation and emission filters used were 380 and $470 \mathrm{~nm}$ respectively for MCB, and 495 and $550 \mathrm{~nm}$ respectively for DOX. Representative fluorescence micrographs at magnification x400 are presented.

Table I. Effect of RALBP1 overexpression and anti-RALBP1 IgG on sensitivity of drugs in K562 cells.

\begin{tabular}{|c|c|c|c|c|c|c|c|c|c|c|c|}
\hline \multirow[b]{2}{*}{ Drug } & \multicolumn{4}{|c|}{ Pre-immune IgG } & \multicolumn{4}{|c|}{ Anti-RALBP1 IgG } & \multicolumn{3}{|c|}{$\begin{array}{c}\text { Ratio } \mathrm{IC}_{50}(\mathrm{RALBP} 1 / \\
\text { pre-immune } \mathrm{IgG})\end{array}$} \\
\hline & Control & Vector & RALBP1 & $\begin{array}{c}\text { Ratio } \mathrm{IC}_{50} \\
\text { (RALBP1/ } \\
\text { vector) }\end{array}$ & Control & Vector & RALBP1 & $\begin{array}{c}\text { Ratio } \mathrm{IC}_{50} \\
\text { (RALBP1/ } \\
\text { vector) }\end{array}$ & Control & Vector & RALBP1 \\
\hline DOX & $90 \pm 8$ & $80 \pm 11$ & $410 \pm 38$ & 5.1 & $38 \pm 6$ & $36 \pm 4$ & $47 \pm 4$ & 1.3 & 0.4 & 0.5 & 0.1 \\
\hline DAU & $220 \pm 28$ & $280 \pm 26$ & $550 \pm 56$ & 2.0 & $80 \pm 14$ & $90 \pm 11$ & $120 \pm 9$ & 1.3 & 0.4 & 0.3 & 0.2 \\
\hline VCR & $40 \pm 5$ & $38 \pm 3$ & $97 \pm 6$ & 2.6 & $17 \pm 2$ & $18 \pm 1$ & $22 \pm 3$ & 1.2 & 0.4 & 0.5 & 0.2 \\
\hline VLB & $10 \pm 1$ & $10 \pm 1$ & $24 \pm 3$ & 2.4 & $4 \pm 1$ & $5 \pm 1$ & $7 \pm 1$ & 1.4 & 0.4 & 0.5 & 0.3 \\
\hline VRL & $4 \pm 1$ & $4 \pm 1$ & $11 \pm 1$ & 3.1 & $2 \pm 0.2$ & $2 \pm 0.2$ & $2 \pm 0.3$ & 1.3 & 0.4 & 0.4 & 0.2 \\
\hline MMC & $25 \pm 3$ & $28 \pm 3$ & $68 \pm 7$ & 2.4 & $14 \pm 1$ & $14 \pm 1$ & $19 \pm 2$ & 1.4 & 0.6 & 0.5 & 0.3 \\
\hline MEL & $6,000 \pm 770$ & $5,000 \pm 330$ & $22,000 \pm 1950$ & 4.4 & $2,500 \pm 220$ & $2,500 \pm 220$ & $3,500 \pm 510$ & 1.4 & 0.4 & 0.5 & 0.2 \\
\hline CDDP & $3,500 \pm 560$ & $4,000 \pm 530$ & $9,000 \pm 825$ & 2.3 & $2,000 \pm 420$ & $2,500 \pm 340$ & $3,500 \pm 470$ & 1.4 & 0.6 & 0.6 & 0.4 \\
\hline
\end{tabular}

The $\mathrm{IC}_{50}$ values in nanomolar are presented as mean $\pm \mathrm{SD}$ from three separate determinations with eight replicates each ( $\left.\mathrm{n}=24\right)$. Antibody concentration (either pre-immune or anti-RALBP1 IgG) is $37 \mu \mathrm{g} / \mathrm{ml}$.

MCB and DOX and examined with dual-excitation at 380 and $470 \mathrm{~nm}$ and emission at 495 and $550 \mathrm{~nm}$ showed the relatively greater accumulation of DOX in the nuclear compartment.
RALBP1 expression reduced the overall uptake of both drugs, and this effect was particularly apparent for DOX in the membrane compartment (Fig. 2). 
Multi-drug resistance conferred by RALBPI overexpression. Cytotoxicity assays were performed to examine the effects of RALBP1 transfection on anthracyclines (DOX, DAU), vinca alkaloids (VCR, VBL, and VRL), alkylating agents (MEL, MMC) and platinum coordinate (CDDP). The wild-type, empty-vector transfected, and RALBP1-transfected cell $\mathrm{IC}_{50}$ was determined in cells growing in log phase $24 \mathrm{~h}$ after addition of pre-immune IgG or anti-RALBP1 IgG (Table I). Whereas empty vector transfection had little or no effect, RALBP1 overexpression yielded 2-5-fold resistance to the cytotoxic effects for all drugs tested. Anti-RALBP1 IgG reduced the $\mathrm{IC}_{50}$ of all cell lines, and caused reversion of resistance to the drugs in the RALBP1-overexpressing cells to near that seen in the wild-type or empty-vector transfected.

Significance. The results of the present study show for the first time that overexpression of RALBP1 results in a broadly drug-resistant phenotype in K562 human erythroleukemia cells. Resistance encompassed the classical Pgp substrates (vinca and anthracyclines), as well as GS-E forming drugs (MMC, CDDP, MEL) known to be transported by ABCC1, ABCG2, and other MRP-related transporters $(5-8,33)$. The resistance was due to decreased overall cellular drug-accumulation caused by greater efflux. The specific nature of resistance was demonstrated by reversal using anti-RALBP1 IgG which has been shown previously to bind to a cell-surface epitope of RALBP1 (aa 171-185) and inhibit the transport activity of RALBP1 (34). The differential ability of RALBP1 to mediate greater resistance to DOX than DAU suggests a possible reason for the greater efficacy of DAU in leukemia as compared with DOX. The ability of RALBP1 overexpressing cells to preferentially lower nuclear accumulation of DOX suggests that this transporter is operational also at the nuclear membrane. In this context, it should be noted that our recent study shows that RALBP1 mRNA depletion causes cancerspecific apoptosis in a number of malignant cell lines, including K562 (22). The present findings highlight the possibility of using RALBP1 as a target not only to reverse multidrug-resistance, but also to directly mediate apoptosis. Pathological and clinical correlations are necessary to determine whether RALBP1 plays a role in clinical multidrugresistance in leukemia.

\section{Acknowledgements}

This work was supported in part by USPHS grant CA 77495 and CA104661 (S.A.) and the Cancer Research Foundation of North Texas (S.S., S.Y. and S.A.). We thank Dr Sophia Passy and Chad Larson, Biology Department, University of Texas at Arlington, Arlington, TX, for helping and providing Confocal Laser Microscope and LSM Image Analysis software, supported by the National Science Foundation grant 0215852 .

\section{References}

1. Nooter K and Sonneveld P: Clinical relevance of P-glycoprotein expression in haematological malignancies. Leuk Res 18: 233-243, 1994.

2. Leith C: Multidrug resistance in leukemia. Curr Opin Hematol 5: 287-291, 1998.
3. Sharma R, Awasthi YC, Yang Y, Sharma A, Singhal SS and Awasthi S: Energy dependent transport of xenobiotics and its relevance to multidrug resistance. Curr Cancer Drug Targets 3: 89-107, 2003.

4. Takara K, Sakaeda T and Okumura K: An update on overcoming MDR1-mediated multidrug resistance in cancer chemotherapy. Curr Pharm Des 12: 273-286, 2006.

5. Gottesman MM and Pastan I: Biochemistry of multidrug resistance mediated by the multidrug transporter. Annu Rev Biochem 62: 385-427, 1993.

6. Szakacs G, Paterson JK, Ludwig JA, Booth-Genthe C and Gottesman MM: Targeting multidrug resistance in cancer. Nat Rev Drug Discov 5: 219-234, 2006.

7. Borst P, Evers R, Kool M and Wijnholds J: The multidrug resistance protein family. Biochim Biophys Acta 1461: 347-357, 1999.

8. Haimeur A, Conseil G, Deeley RG and Cole SP: The MRPrelated and BCRP/ABCG2 multidrug resistance proteins: biology, substrate specificity and regulation. Curr Drug Metab 5: 21-53, 2004.

9. Jakoby WB: The glutathione S-transferases: a group of multifunctional detoxification proteins. Adv Enzymol Relat Areas Mol Biol 46: 383-414, 1978.

10. Hayes JD and Pulford DJ: The glutathione S-transferase supergene family: regulation of GST and the contribution of the isoenzymes to cancer chemoprotection and drug resistance. Crit Rev Biochem Mol Biol 30: 445-600, 1995.

11. Sharma R, Brown D, Awasthi S, et al: Transfection with 4hydroxynonenal-metabolizing glutathione S-transferase isozymes leads to phenotypic transformation and immortalization of adherent cells. Eur J Biochem 271: 1690-1701, 2004.

12. Awasthi YC, Yang Y, Tiwari NK, Patrick B, Sharma A, Li J and Awasthi S: Regulation of 4-hydroxynonenal-mediated signaling by glutathione S-transferases. Free Radic Biol Med 37: 607-619, 2004.

13. Ramana KV, Bhatnagar A, Srivastava S, Yadav UC, Awasthi S, Awasthi YC and Srivastava SK: Mitogenic responses of vascular smooth muscle cells to lipid peroxidation-derived aldehyde 4-hydroxy-trans-2-nonenal (HNE): role of aldose reductase-catalyzed reduction of the HNE-glutathione conjugates in regulating cell growth. J Biol Chem 281: 17652-17660, 2006.

14. Awasthi S, Singhal SS, Yadav S, et al: RLIP76 is a major determinant of radiation sensitivity. Cancer Res 65: 6022-6028, 2005.

15. Awasthi S, Cheng J, Singhal SS, et al: Novel function of human RLIP76: ATP-dependent transport of glutathione conjugates and doxorubicin. Biochemistry 39: 9327-9334, 2000 .

16. Awasthi S, Sharma R, Singhal SS, Zimniak P and Awasthi YC: RLIP76, a novel transporter catalyzing ATP-dependent efflux of xenobiotics. Drug Metab Dispos 30: 1300-1310, 2002.

17. Awasthi S, Singhal SS, Sharma R, Zimniak P and Awasthi YC: Transport of glutathione conjugates and chemotherapeutic drugs by RLIP76 (RALBP1): a novel link between G-protein and tyrosine kinase signaling and drug resistance. Int J Cancer 106: 635-646, 2003.

18. Sharma R, Singhal SS, Wickramrachchi D, Awasthi YC and Awasthi S: RLIP76 (RALBP1) mediated transport of leukotriene C4 (LTC4) in cancer cells: implications in drug-resistance. Int J Cancer 112: 934-942, 2004.

19. Stuckler D, Singhal J, Singhal SS, Yadav S, Awasthi YC and Awasthi S: RLIP76 transports vinorelbine and mediates drug resistance in non-small cell lung cancer. Cancer Res 65: 991-998, 2005.

20. Singhal SS, Yadav S, Singhal J, Zajac E, Awasthi YC and Awasthi S: Depletion of RLIP76 sensitizes lung cancer cells to doxorubicin. Biochem Pharmacol 70: 481-488, 2005.

21. Singhal SS, Wickramarachchi D, Singhal J, Yadav S, Awasthi YC and Awasthi S: Determination of differential doxorubicin sensitivity between SCLC and NSCLC. FEBS Lett 580: 2258-2264, 2006

22. Singhal SS, Awasthi YC and Awasthi S: Complete regression of melanoma in a murine model by RLIP76 depletion. Cancer Res 66: 2354-2360, 2006.

23. Awasthi S, Singhal SS, Srivastava SK, et al: Adenosine triphosphate-dependent transport of doxorubicin, daunomycin, and vinblastine in human tissues by a mechanism distinct from the P-glycoprotein. J Clin Invest 93: 958-965, 1994. 
24. Awasthi S, Singhal SS, Pandya U, Gopal S, Zimniak P, Singh SV and Awasthi YC: ATP-dependent colchicine transport by human erythrocyte glutathione conjugate transporter. Toxicol Appl Pharmacol 155: 215-226, 1999.

25. Ikeda M, Ishida O, Hinoi T, Kishida S and Kikuchi A: Identification and characterization of a novel protein interacting with Ral-binding protein 1, a putative effector protein of Ral. J Biol Chem 273: 814-821, 1998 .

26. Rosse C, L'hoste S, Offner N, Picard A and Camonis J: RLIP, an effector of the Ral GTPases, is a platform for Cdk1 to phosphorylate epsin during the switch off of endocytosis in mitosis. J Biol Chem 278: 30597-30604, 2003.

27. $\mathrm{Hu} \mathrm{Y}$ and Mivechi NF: HSF-1 interacts with Ral-binding protein 1 in a stress-responsive, multiprotein complex with HSP90 in vivo. J Biol Chem 278: 17299-17306, 2003.

28. Singhal SS and Awasthi S: Glutathione-conjugate transport and stress-response signaling: role of RLIP76. In: Toxicology of Glutathione S-Transferases. Awasthi YC (ed). CRC Press, Boca Raton, FL, pp231-256, 2006.
29. Singhal SS, Singhal J, Sharma R, Singh SV, Zimniak P, Awasthi YC and Awasthi S: Role of RLIP76 in lung cancer doxorubicin resistance: I. The ATPase activity of RLIP76 correlates with doxorubicin and 4-hydroxynonenal resistance in lung cancer cells. Int J Oncol 22: 365-375, 2003.

30. Awasthi S, Singhal SS, He NG, et al: Modulation of doxorubicin cytotoxicity by ethacrynic acid. Int J Cancer 68: 333-339, 1996.

31. Briviba K, Fraser G, Sies H and Ketterer B: Distribution of the monochlorobimane-glutathione conjugate between nucleus and cytosol in isolated hepatocytes. Biochem J 294: 631-633, 1993.

32. Millis KK, Lesko SA and Gamcsik MP: Formation, intracellular distribution and efflux of glutathione-bimane conjugates in drug-sensitive and -resistant MCF-7 cells. Cancer Chemother Pharmacol 40: 101-111, 1997.

33. Sarkadi B, Ozvegy-Laczka C, Nemet K and Varadi A: ABCG2 - a transporter for all seasons. FEBS Lett 567: 116-120, 2004.

34. Yadav S, Singhal SS, Singhal J, et al: Identification of membrane anchoring domains of RLIP76 using deletion mutants analyses. Biochemistry 43: 16243-16253, 2004 